\title{
Evaluación de la dimensión científico-clínica del cuidado en el personal de enfermería*
}

\author{
Evaluation of the scientific-clinical dimension of care in the nursing workers \\ Avaliação da dimensão científico-clínica do cuidado na equipe de enfermagem
}

Angela María Henao-Castaño ${ }^{a}$

Universidad Nacional de Colombia, Colombia

DOI: https://doi.org/10.11144/Javeriana.ie23.edcc

angmhenaocas@unal.edu.co

ORCID: https://orcid.org/0000-0003-4203-0016

Recibido: 03 Marzo 2021

Myriam Lucía Avila

Aceptado: 07 Septiembre 2021

Hospital Militar Central, Colombia

Publicado: 30 Diciembre 2021

ORCID: https://orcid.org/0000-0002-7101-8836

Soraya Ramos Hernández

Hospital Militar Central, Colombia

ORCID: https://orcid.org/0000-0003-2250-3584

Sandra Rocío Guaqueta-Parada

Universidad Nacional de Colombia, Colombia

ORCID: https://orcid.org/0000-0002-2354-7493

Martha Cecilia Triana-Restrepo

Universidad Nacional de Colombia, Colombia

ORCID: https://orcid.org/0000-0002-9991-7999

\section{Resumen:}

La evaluación de la dimensión científico-clínica del cuidado de enfermería basado en el modelo HANC en la Unidad de Cuidado Intensivo (VDCC-UCI) permitió medir la calidad del cuidado en los procesos de atención de enfermería en la Unidad de Cuidado Intensivo. Objetivo: Describir la dimensión científico-clínica del cuidado de enfermería en la Unidad de Cuidado Intensivo, comparando entre enfermeras de instituciones públicas y privadas. Método: Estudio descriptivo de tipo transversal realizado entre de julio y diciembre de 2019. Participaron 186 enfermeros que trabajan en la Unidad de Cuidado Intensivo de instituciones públicas y privadas del país. Los datos fueron recolectados con el instrumento de la dimensión científico-clínica. Resultados: Los participantes, en su mayoría mujeres $(74 \%)$ y hombres ( $48 \%$ ), contaban con una especialización (75\%) y con experiencia profesional más de diez años; el tipo de contratación más común fue el de término indefinido. Se encontraron diferencias estadísticamente significativas en 18 de los 46 ítems entre las respuestas de enfermeras de instituciones públicas en comparación con las de instituciones privadas. Conclusiones: La evaluación de la dimensión científico-clínica mostró que algunas etapas del proceso de atención de enfermería se llevan a cabo de manera más rigurosa y completa por parte de las enfermeras de instituciones privadas, mientras las etapas del proceso administrativo e investigativo implementadas por enfermeras de entidades públicas y privadas son similares.

Palabras clave: cuidados críticos, enfermería, atención en enfermería.

\section{Abstract:}

The evaluation of the scientific-clinical of nursing care based on the HANC model at intensive care unit (VDCC-ICU) enabled to measure the care quality in the nursing care processes at the ICU. Objective: To describe the scientific-clinical dimension of the nursing care at the ICU compering nurses from public centers to private center. Methods: It is a cross-sectional descriptive study conducted from July to December 2019. A total of 186 nurses who work at the ICU in both public and private centers in our country took part in the study. Data were gathered through a scientific-clinical instrument. Results: A $75 \%$ of the participants (women) and $48 \%$ (men) had earned a specialization degree and $75 \%$ had a work experience longer than 10 years. The most frequent

Notas de autor

\footnotetext{
a Autora de correspondencia: angmhenaocas@unal.edu.co
} 
hiring was as under an indefinite term contract. Sadistically significant differences were found in 18 out of 46 items regarding the answers by nurses form public and private centers. Conclusions: The evaluation of the scientific-clinical showed the some stages in the nursing care process are carried out more rigorously and complete by nurses from private centers, while the administrative and research stages of the process are carried out quite similarly by both public and private nurses.

Keywords: critical care, nursing, nursing care.

\section{Resumo:}

A avaliação da dimensão científico-clínica do cuidado de enfermagem baseado no modelo HANC na Unidade de Terapia Intensiva (VDCC-UTI) possibilitou mensurar a qualidade do cuidado nos processos de atendimento de enfermagem na Unidade de Terapia Intensiva. Objetivo: Descrever a dimensão científico-clínica do cuidado de enfermagem na Unidade de Terapia Intensiva, comparando enfermeiros de instituições públicas e privadas. Método: Estudo descritivo transversal realizado entre julho e dezembro de 2019. Participaram 186 enfermeiros da Unidade de Terapia Intensiva de instituiçóes públicas e privadas do país. Os dados foram coletados com o instrumento da dimensão científico-clínica. Resultados: os participantes, em sua maioria mulheres (74\%) e homens 48\%, possuíam especialização (75\%) e experiência profissional superior a 10 anos; o tipo de contrato mais comum foi o de prazo indeterminado. Encontraram-se diferenças estatisticamente significativas em 18 dos 46 itens entre as respostas de enfermeiros de instituições públicas em comparação com as de instituições privadas. Conclusões: A avaliação da dimensão científico-clínica mostrou que algumas etapas do processo de atenção de enfermagem são realizadas de maneira mais rigorosa e completa por parte dos enfermeiros de instituições privadas, enquanto as etapas do processo administrativo e investigativo implementadas por enfermeiros de entidades públicas e privadas são semelhantes.

Palavras-chave: cuidados críticos, enfermagem, atendimento em enfermagem.

\section{Introducción}

El paciente en situación crítica de salud requiere, para su cuidado y tratamiento en la Unidad de Cuidado Intensivo (UCI), de servicios altamente organizados como la atención médica y de enfermería especializada, que incluyen la utilización de tecnología avanzada para realizar seguimiento, monitoreo y soporte fisiológico, con el objetivo de preservar su vida y favorecer su recuperación (1).

Según las características del paciente en situación crítica de salud, la enfermera juega un papel muy importante en su cuidado, pues esta constantemente identifica, analiza, evalúa, interpreta e interviene en las diferentes situaciones que involucran al paciente y la familia; en este sentido, desde el grupo de investigación de urgencias y cuidado crítico, se ha propuesto un modelo de cuidado de enfermería para pacientes en situación crítica de salud en la UCI denominado HANC, modelo que toma su nombre de cuatro dimensiones derivadas del análisis de los referentes teóricos y los patrones de conocimiento en enfermería: $\mathrm{H}$, humanización del cuidado; $\mathrm{A}$, ayuda al paciente crítico hospitalizado; $\mathrm{N}$, necesidades satisfechas; y $\mathrm{C}$, cuidado de calidad (2).

La dimensión científico-clínica se refiere al quehacer de esta disciplina mediante la aplicación de los conocimientos científicos y tecnológicos en el escenario de la UCI; la aplicación de esta dimensión implica un proceso analítico, reflexivo y crítico que busca recuperar o conservar el estado de salud del individuo, identificando sus necesidades, determinando un plan a seguir y evaluando el logro de los resultados esperados, partiendo de tres categorías: el proceso de atención de enfermería, el proceso administrativo y el proceso investigativo (2).

El proceso de atención de enfermería es la aplicación del método científico en el saber y el quehacer de enfermería, en el cual la enfermera valora, realiza un juicio clínico, planea y ejecuta intervenciones, teniendo en cuenta sus habilidades relacionales y de uso de la tecnología; además, evalúa el trabajo realizado determinado por el grado de satisfacción del paciente y su familia (3).

El proceso administrativo se definió como la aplicación de un juicio profesional en la planificación, organización, motivación, ejecución y control de la provisión de cuidados eficaces e integrales que aseguren la continuidad de la atención (2). 
El proceso investigativo permite generar conocimiento y construir la evidencia de enfermería, con el objetivo de sustentar las intervenciones, proporcionar soluciones y mejoras en la calidad del cuidado (4).

De acuerdo con lo anterior, la dimensión científico-clínica de enfermería involucra el saber y el saber-hacer fundamentado en conocimientos propios de la disciplina, a través de la articulación, de la fundamentación teórica, las habilidades y la práctica basada en evidencia (5).

En cuanto al desarrollo de este tema de estudio, se evidenció que la investigación en el ámbito colombiano es poca y se relaciona con la formulación de planes de cuidado para diferentes pacientes en UCI, así como con la identificación del proceso de atención o de los procesos investigativos o administrativos de manera separada.

Un estudio que buscaba describir los factores relacionados con la aplicación del proceso de enfermería en la UCI (6) reportó que las enfermeras realizan la valoración y las intervenciones según los protocolos de la UCI; también planean el cuidado de acuerdo con el diagnóstico médico y, en menor medida, formulan diagnósticos de enfermería y evalúan.

La aplicación del proceso de enfermería en la UCI reportado en la literatura (7) mostró que este incluye intervenciones asistenciales y registros, y es utilizado como una herramienta de planificación y gestión del cuidado.

En cuanto al proceso administrativo, la enfermera gestiona recursos físicos materiales y humanos, coordina, ejecuta y evalúa las actividades de enfermería. Varias investigaciones (8) (9) dirigen sus análisis a la interpretación sobre la manera en que competencias tales como el liderazgo de la enfermera (10), el trabajo en equipo (11) y las labores administrativas puntuales, es decir, rondas de enfermería, actualización de historias clínicas, revisión de órdenes médicas, coordinación de actividades, elaboración de notas de cargo y de pedidos (12) inciden en la definición de este proceso, pero no involucran la totalidad del cuidado de enfermería que refleja la dimensión científico-clínica definida para el modelo HANC.

En relación al proceso de investigación en enfermería, la literatura reporta que existen dos dimensiones de análisis: la primera, respecto a los aportes a la gestión del cuidado en la UCI, con investigaciones sobre seguridad del paciente, indicadores de calidad, competencias de la enfermera y aportes a la disciplina; y la segunda, referente a la práctica especializada en la UCI, con investigaciones sobre comunicación enfermerapaciente, trabajo en equipo, modelos de atención y guías de práctica clínica (11).

No se encontraron estudios que muestren si la enfermera aplica los procesos de cuidado de enfermería, administrativos e investigativos de manera integrada en el servicio en la UCI, tal como se ha formulado desde los planteamientos del modelo HANC, en el cual es ideal la integración de los tres procesos.

Teniendo en cuenta lo mencionado hasta el momento, el objetivo de este estudio fue describir la dimensión científico-clínica del cuidado de enfermería en la UCI, en instituciones prestadoras de salud públicas y privadas, mediante la aplicación del instrumento de evaluación basado en el modelo HANC en la unidad de cuidado intensivo (VDCC-UCI).

\section{Método}

Estudio descriptivo de tipo cuantitativo y de corte transversal, desarrollado durante los meses de julio a noviembre de 2019.

Para la medición de la dimensión científico-clínica se utilizó el instrumento VDCC-UCI, compuesto por 46 ítems, estructurados según las fases del proceso de atención de enfermería (22 ítems), el proceso administrativo (21 ítems) y el proceso investigativo (3 ítems), y calificados con una escala de Likert que oscila entre 0 (nunca) y 3 (siempre).

Las puntuaciones altas obtenidas con el instrumento indican que la enfermera lleva a cabo sus acciones, sustentadas desde un saber científico, utilizando las fases de los tres procesos, las cuales le permiten brindar el cuidado a partir de la identificación de las necesidades de ayuda e insatisfechas, que promuevan la formulación 
de planes de cuidado. El instrumento cuenta con una validez facial, de contenido y de constructo con un alfa de Cronbach de 0,93 (13).

La muestra estuvo constituida por 186 enfermeros y enfermeras que laboran en UCI de hospitales públicos y privados del país. Se utilizó un muestreo intencional, el cual se calculó teniendo en cuenta 8 participantes por 22, que es el número de ítems de la dimensión del proceso de atención de enfermería del instrumento utilizado, teniendo en cuenta que es la dimensión con mayor número de ítems, según (14).

El perfil de selección de los participantes era: enfermera o enfermero que haya trabajado en la UCI para adultos de una institución de tercer nivel por un periodo mayor a seis meses. Estas personas fueron contactadas por el grupo de investigación para presentarles la información del proyecto e invitarlos a hacer parte del mismo; con base en su respuesta, dieron su autorización mediante el consentimiento informado.

Para el análisis de datos se aplicó estadística descriptiva e inferencial; para la caracterización de los participantes se calcularon correlaciones. La distribución de las variables fue normal, de acuerdo con el test de Kolgomorov Smirnov, por lo tanto, se llevó a cabo el coeficiente de Pearson (r) y Chi cuadrado de Pearson, y para variables dicotómicas se reportaron como valores . significativos aquellos menores a 0,05. Se utilizó el programa STATA versión 21.0, considerando las variables demográficas (edad, género y nivel educativo) y laborales (tipo de institución donde trabaja, experiencia y tipo de contrato).

El estudio cumplió con la legislación vigente para la investigación con seres humanos, es decir, con la declaración de Helsinki de la Asamblea Médica Mundial, la Resolución 8430 de 1993 y la Ley 266 de 1966. El estudio fue calificado con riesgo mínimo y contó con el aval ético del Comité de la Facultad de Enfermería de la Universidad Nacional.

\section{Resultados}

Al evaluar comparativamente las variables sociodemográficas (tabla 1), se encontró que las enfermeras de las instituciones privadas eran cuatro años más jóvenes que las de las instituciones públicas; esto representó una diferencia estadísticamente significativa $(p=0,0011)$. A su vez, se observó que el 64,7 \% de las enfermeras de instituciones privadas presentaban un mayor nivel educativo, pues contaban con formación de posgrado, mientras que el $66,3 \%$ de las enfermeras de instituciones públicas solo tenían pregrado; esta diferencia también fue estadísticamente significativa $(p=0,000)$.

Por otra parte, el análisis mostró una relación significativa respecto a la experiencia laboral y al tipo de institución, pues más de la cuarta parte de las enfermeras de instituciones privadas $(25,9 \%)$ tenían entre 7 y 9 años de experiencia, mientras que en las instituciones públicas esta cifra fue apenas del 11,9\%. En ese mismo sentido, se encontró que un 20,8 \% de enfermeras tiene experiencia de 4 a 6 años trabajando en instituciones públicas comparadas con el $12,9 \%$ que trabajan en instituciones privadas con este mismo rango de experiencia laboral.

Con respecto al tipo de contrato, se evidenció que el 71,8 \% de las enfermeras de instituciones privadas tienen contrato a término indefinido, mientras que el 41,6\% de las enfermeras de instituciones públicas tiene contrato por prestación de servicios. 
TABLA 1

Variables sociodemográficas según tipo de institución

\begin{tabular}{|c|c|c|c|c|c|c|}
\hline \multirow{2}{*}{\begin{tabular}{l|}
\multicolumn{1}{c|}{ Ítems } \\
Edad (mediana, \\
rango)
\end{tabular}} & \multicolumn{2}{|c|}{ Privadas $(n=85)$} & \multicolumn{2}{|c|}{ Públicas $(n=101)$} & \multirow{2}{*}{$\begin{array}{l}\begin{array}{c}\text { Total } \\
(\mathbf{n}=\mathbf{1 8 6})\end{array} \\
37(22- \\
58) \\
\end{array}$} & \multirow{2}{*}{$\begin{array}{r}\text { Prueba } \\
0,0011^{\mathrm{a}} \dagger\end{array}$} \\
\hline & 34 & $22-56$ & 38 & $23-58$ & & \\
\hline \multicolumn{7}{|l|}{ Género } \\
\hline Masculino & 24 & $28,2 \%$ & 24 & $23,8 \%$ & $48(26)$ & \multirow{2}{*}{$0,487^{b}$} \\
\hline Femenino & 61 & $71,8 \%$ & 77 & $76,2 \%$ & $138(74)$ & \\
\hline \multicolumn{7}{|l|}{$\begin{array}{l}\text { Nivel } \\
\text { académico }\end{array}$} \\
\hline Pregrado & 30 & $35,30 \%$ & 67 & $66,30 \%$ & 97 & \multirow{4}{*}{$0,000^{c} \dagger$} \\
\hline Especialización & 40 & $47,10 \%$ & 29 & $28,70 \%$ & 69 & \\
\hline Maestría & 14 & $16,50 \%$ & 5 & $5,00 \%$ & 19 & \\
\hline Doctorado & 1 & $1,20 \%$ & 0 & $0,00 \%$ & 1 & \\
\hline \multicolumn{7}{|l|}{$\begin{array}{l}\text { Experiencia } \\
\text { laboral }\end{array}$} \\
\hline 1-3 años & 12 & $14,10 \%$ & 8 & $7,90 \%$ & 20 & \multirow{4}{*}{$0,023^{b}+$} \\
\hline $4-6$ & 11 & $12,90 \%$ & 21 & $20,80 \%$ & 32 & \\
\hline $7-9$ & 22 & $25,90 \%$ & 12 & $11,90 \%$ & 34 & \\
\hline$>=10$ años & 40 & $47,10 \%$ & 60 & $59,40 \%$ & 100 & \\
\hline \multicolumn{7}{|l|}{$\begin{array}{l}\text { Tipo de } \\
\text { contrato }\end{array}$} \\
\hline $\begin{array}{l}\text { Término } \\
\text { indefinido }\end{array}$ & 61 & $71,80 \%$ & 37 & $36,60 \%$ & 98 & \multirow{3}{*}{$0,000^{b} \uparrow$} \\
\hline Término fijo & 13 & $15,30 \%$ & 22 & $21,80 \%$ & 35 & \\
\hline $\begin{array}{l}\text { Prestación de } \\
\text { servicios }\end{array}$ & 11 & $12,90 \%$ & 42 & $41,60 \%$ & 53 & \\
\hline
\end{tabular}

a; † significancia estadística: $\mathrm{p}<0,05$

Fuente: Los indicadores han sido tomados del estudio de 2019 y el diseño de la tabla es una elaboración propia.

Tal y como se muestra a continuación, el análisis comparativo de los resultados obtenidos a partir de la aplicación del instrumento, teniendo en cuenta el tipo de institución en donde trabajan las enfermeras, evidencia diferencias estadísticamente significativas en 18 de los 46 ítems; las respuestas que tuvieron mayor frecuencia se presentan en la tabla 2.

En cuanto a la categoría de aplicación del proceso de enfermería se evidenció que el 86,2\% de las enfermeras de instituciones públicas, siempre o casi siempre, se presentan al paciente con nombre completo y cargo antes de comenzar la valoración (ítem 1), mientras que en las instituciones privadas se observó que, siempre o casi siempre, el 97,6\% de las enfermeras se presentan $(p=0,009)$. Por otro lado, se encontró que el total del personal de enfermería de instituciones privadas siempre o casi siempre establece prioridad entre los diagnósticos de enfermería identificados (ítem 7); por otra parte, en instituciones públicas solo el $91,1 \%$ de las enfermeras siempre o casi siempre realizan esta acción $(p=0,023)$.

Asimismo, el 90,6 \% del personal de enfermería de la institución privada siempre o casi siempre formula diagnósticos de riesgo en el paciente (ítem 8); en la institución pública, esta acción fue realizada en una menor proporción de enfermeras $(70,3 \%)$, siendo un dato estadísticamente significativo $(p=0,004)$. También se evidenció que el $87,1 \%$ de las enfermeras de instituciones privadas elaboran, casi siempre o siempre, el plan de cuidado de enfermería diariamente, según estado del paciente (ítem 10); sin embargo, en la institución pública el 32,7 \% de las enfermeras casi nunca o nunca elaboran el plan de cuidado diario $(p=0,000)$.

Otro de los hallazgos fue que el 63,5\% del personal de enfermería en instituciones privadas siempre establece priorización para las intervenciones en la ejecución del plan de cuidado (ítem 11); en cambio, en la institución pública, esta acción fue realizada, regularmente, en una menor proporción de enfermeras $(39,6$ $\%)$, mientras que el $8 \%$ que casi nunca la realizan $(p=0,004)$.

En el ítem 21 se observó que el 97,6 \% del personal de enfermería en institución privada, siempre o casi siempre, evalúa los avances positivos del estado de salud del paciente, algo que ocurre en proporciones similares en la institución pública, donde se encontró que el 93,1 \% de la población entrevistada realizaba dicha evaluación; este indicador es estadísticamente significativo $(p=0,044)$.

El nivel de satisfacción del paciente con el cuidado de enfermería recibido (ítem 22) fue evaluado siempre o casi siempre por el 87,1 \% del personal de enfermería de las instituciones privadas, mientras que el 71,3\% de las enfermeras de instituciones públicas casi siempre o siempre realizan esta evaluación $(p=0,009)$. En ese 
mismo sentido, la evaluación sobre el nivel de satisfacción de la familia con el cuidado de enfermería ofrecido al paciente (ítem 23) fue realizado siempre o casi siempre por el 71,8\% del personal de enfermería de institución privada; en cambio, el personal del sector público realiza esta evaluación en menor proporción (40,6 \%), diferencia que también fue estadísticamente significativa $(p=0,000)$.

En cuanto a la categoría de aplicación del proceso administrativo, el 91,8 \% del personal de instituciones privadas aplica siempre o casi siempre las escalas valorativas de dependencia y/o riesgo en el paciente (ítem 28); en el sector público, esta actividad es realizada en un $77,2 \%$, siendo estadísticamente significativo $(p=0,023)$. Respecto al ítem 29 , se evidenció que un alto porcentaje de enfermeras de institución privada (96,7 \%) realiza la asignación al personal a cargo siempre o casi siempre; por otra parte, en la institución pública se observó que el 74,3\% de las enfermeras realizan la asignación correspondiente $(p=0,000)$.

Ahora bien, las habilidades y destrezas del personal a cargo son conocidas por todo el personal de enfermería del sector privado (100\%), mientras que en el sector público esta variable fue del $96 \%$, es decir, el $4 \%$ restante casi nunca conoce dichas habilidades y destrezas $(p=0,021)$. En cuanto al ítem 32, se encontró que el 70,6\% de las enfermeras de institución privada casi siempre o siempre entregan el folleto informativo a la familia, mientras que solo el $43,6 \%$ de las enfermeras del sector público entregan dicho folleto $(p=0,002)$.

A su vez, la supervisión de las acciones del personal a cargo en cada turno (ítem 37) fue realizada por el 95,3 $\%$ de las enfermeras de institución privada; en la institución pública, este porcentaje fue del $93,1 \%(p=0,000)$. En el mismo sentido, la detección de fallas y corrección de errores de las acciones supervisadas (ítem 38) se realiza en un $65,9 \%$ en personal del sector privado, mientras que el personal del sector público lo realiza en un $42,6 \%(p=0,005)$.

En lo que respecta a las medidas de confort ambiental para el paciente en la UCI (alarmas, mantas térmicas, aire acondicionado, luz, ruido, voz, etc.) (ítem 39), estas son utilizadas casi siempre o siempre por el $98,8 \%$ del personal de enfermería de la institución privada; por el contrario, en la institución pública, aproximadamente, el $88,1 \%$ de las enfermeras utilizan medidas de confort ambiental $(p=0,000)$. La organización de las actividades que preservan los periodos de descanso del paciente (ítem 41) fue mayor en el personal de enfermería del sector privado, comparado con el personal del sector público $(94,1 \%$ y $89,1 \%$ respectivamente), siendo esta una diferencia significativa $(p=0,008)$.

Con relación a la categoría de aplicación del proceso investigativo, en el personal de enfermería de instituciones privadas las situaciones problema de la práctica (ítem 44) son identificadas para formular un proyecto investigativo en un $55,3 \%$, mientras que en el sector público el $68,3 \%$ nunca o casi nunca identifican propuestas de investigación a partir de los problemas de la práctica $(p=0,005)$. En consecuencia, la participación en el desarrollo de proyectos de investigación (ítem 45) es baja; sin embargo, esta participación en el personal del sector privado es superior con respecto al sector público (38,8 \% y 20,8 \% respectivamente), consolidándose como una diferencia estadísticamente significativa $(p=0,008)$. 
TABLA 2

Resultados instrumento dimensión científico-clínica

\begin{tabular}{|c|c|c|c|c|c|c|c|}
\hline \multicolumn{8}{|c|}{ Instrumento dimensión científico-clínica } \\
\hline \multirow[b]{2}{*}{ İtems } & \multirow[t]{2}{*}{\begin{tabular}{|c|c|}
$\begin{array}{c}\text { Frecuencia } \\
\text { respuesta }\end{array}$ \\
\end{tabular}} & \multicolumn{2}{|c|}{$\begin{array}{c}\text { Privado } \\
(n=85)\end{array}$} & \multicolumn{2}{|c|}{$\begin{array}{l}\text { Público } \\
(\mathrm{n}=101)\end{array}$} & \multirow{2}{*}{$\begin{array}{c}\frac{\begin{array}{c}\text { Total } \\
(\mathrm{n}=186)\end{array}}{\mathrm{N}} \\
\end{array}$} & \multirow[t]{2}{*}{\begin{tabular}{|c|}
$\begin{array}{c}\text { Prueba } \\
\text { sig }\end{array}$ \\
\end{tabular}} \\
\hline & & $\mathbf{N}$ & $\%$ & $\mathbf{N}$ & $\%$ & & \\
\hline \multicolumn{8}{|c|}{ Proceso de atención de enfermería } \\
\hline Ítem 1 & Siempre & 43 & $51 \%$ & 34 & $34 \%$ & 77 & $\begin{array}{c}0,009 \\
\mathrm{a}^{\dagger}\end{array}$ \\
\hline Ítem 7 & Siempre & 47 & $55 \%$ & 50 & $50 \%$ & 97 & 0,023 \\
\hline Ítem 8 & $\begin{array}{c}\text { Casi } \\
\text { siempre }\end{array}$ & 42 & $49 \%$ & 43 & $43 \%$ & 85 & 0,004 \\
\hline Ítem 10 & Siempre & 43 & $51 \%$ & 22 & $22 \%$ & 65 & 0 \\
\hline Ítem 11 & Siempre & 54 & $64 \%$ & 40 & $40 \%$ & 94 & 0,004 \\
\hline Ítem 21 & Siempre & 48 & $57 \%$ & 40 & $40 \%$ & 88 & 0,044 \\
\hline Ítem 22 & $\begin{array}{c}\text { Casi } \\
\text { siempre }\end{array}$ & 44 & $52 \%$ & 53 & $53 \%$ & 97 & 0,009 \\
\hline Ítem 23 & $\begin{array}{c}\text { Casi } \\
\text { siempre }\end{array}$ & 39 & $46 \%$ & 31 & $31 \%$ & 70 & 0 \\
\hline \multicolumn{8}{|c|}{ Proceso administrativo } \\
\hline Ítem 28 & Siempre & 45 & $53 \%$ & 36 & $36 \%$ & 81 & 0,023 \\
\hline Ítem 29 & Siempre & 53 & $62 \%$ & 42 & $42 \%$ & 95 & 0 \\
\hline Ítem 32 & Siempre & 36 & $42 \%$ & 22 & $22 \%$ & 58 & 0,002 \\
\hline Ítem 37 & Siempre & 55 & $65 \%$ & 36 & $36 \%$ & 91 & 0 \\
\hline Ítem 38 & Siempre & 56 & $66 \%$ & 43 & $43 \%$ & 99 & 0,005 \\
\hline Ítem 39 & Siempre & 54 & $64 \%$ & 38 & $38 \%$ & 92 & 0 \\
\hline Ítem 41 & Siempre & 46 & $54 \%$ & 32 & $32 \%$ & 78 & 0,008 \\
\hline \multicolumn{8}{|c|}{ Proceso investigativo } \\
\hline Ítem 44 & Casi Nunca & 32 & $38 \%$ & 50 & $50 \%$ & 82 & 0,005 \\
\hline Ítem 45 & casi nunca & 39 & $46 \%$ & 47 & $47 \%$ & 86 & 0,008 \\
\hline
\end{tabular}

Fuente: Los indicadores han sido tomados del estudio de 2019 y el diseño de la tabla es una elaboración propia.

\section{Discusión}

En cuanto a los datos sociodemográficos, se encontró un alto porcentaje de profesionales de género femenino con predominio de edad entre 30 a 40 años; esto es consistente con los resultados de otras investigaciones que han estudiado el perfil de los profesionales en esta área (15), y que atribuyen a esta prevalencia femenina la tendencia histórica en la profesión. Llama la atención que la mayoría de estas profesionales no cuentan con educación formal en cuidado crítico, aspecto que se ve compensado con la experiencia mayor de 10 años y que posiblemente se relaciona con los requerimientos de habilitación establecidos hace algunos años, en donde se validaba la experiencia clínica por la formación posgradual (16). Sin embargo, este hallazgo es preocupante teniendo en cuenta la complejidad del paciente que requiere de profesionales con mayor cualificación (17).

En cuanto a los hallazgos relacionados con la aplicación del proceso de atención de enfermería, se identificaron diferencias significativas, mostrando un mayor porcentaje de acciones relacionadas con la formulación de los diagnósticos, priorización, elaboración de planes de cuidado, intervenciones y evaluación por parte de las enfermeras de las instituciones privadas, que puede explicarse por la implementación en estas instituciones del proceso de enfermería de manera sistematizada, gracias a la utilización de un software a través del cual los registros se integran a la historia clínica electrónica y al protocolo de recibo y entrega de turno (18), en contraste con las instituciones que tienen restricciones presupuestales, como las públicas, donde la carencia de tecnología asistencial que facilite la implementación de esta herramienta es predominante (19) (20), datos que aparecen en concordancia con lo reportado por la literatura, en donde se identifica que el proceso de enfermería no se sistematiza y se sustituye por intervenciones rutinarias (21).

Implementar el proceso de enfermería requiere de conocimiento científico, experiencia y habilidades prácticas (22); en este sentido, las diferencias encontradas en cuanto a la formulación y priorización de diagnósticos de enfermería pueden relacionarse con un el nivel de formación de las enfermeras de instituciones privadas, puesto que los procesos de formación posgradual facilitan el análisis crítico y el soporte teórico y conceptual de los profesionales del área (7).

La aplicación del proceso de enfermería presenta múltiples desafíos debido a la diversidad de conceptos que tienen las enfermeras; la falta de tiempo suficiente para su implementación, la diferencia entre lo que se 
enseña y lo que realmente se realiza en la práctica, así como la falta de conocimiento sobre la utilización del mismo son dificultades que derivan en trámites administrativos o actividades centradas en procedimientos (23), que desdibujan el alcance y resultados del proceso de enfermería (21).

Las enfermeras en las instituciones privadas evalúan en mayor proporción la satisfacción del paciente y la familia durante la implementación del proceso de enfermería, lo cual permite hacer seguimiento a las intervenciones, es decir, a la evaluación del cuidado, la eficiencia y la efectividad de las acciones realizadas, de tal manera que se puedan incluir modificaciones de acuerdo a los resultados en la evolución del paciente (24).

El proceso de enfermería computarizado contribuye a una menor carga cognitiva, pues es un sistema de apoyo para la toma de decisiones y una opción confiable para la mejora en la atención en salud que contribuye en la seguridad del paciente y en la atención de enfermería basada en evidencia (25).

En cuanto al proceso administrativo, se muestran diferencias relacionadas con una mayor implementación de etapas del proceso entre las enfermeras del área privada, determinadas por factores asociados a la naturaleza y organización de la institución y al rol del profesional en cuanto a la autonomía, liderazgo, implementación del proceso y formación académica e investigativa (26).

En cuanto a la autonomía profesional, la literatura reporta que esta capacidad se encuentra influenciada por la experiencia que se adquiere en el contacto con la práctica y la asistencia clínica (27); esto explica que la implementación del proceso administrativo en la institución privada esté relacionada con un mayor número de años de experiencia y formación de las profesionales.

Los hallazgos referidos a la aplicación del proceso investigativo muestran que las enfermeras de las instituciones públicas y privadas tienen poca participación en la formulación y desarrollo de proyectos de investigación; esta situación puede relacionarse con la falta de recursos y de apoyos institucionales, al igual que a la falta de tiempo para participar en dichos proyectos (28). Sin embargo, es importante resaltar que el apego a protocolos y guías de atención forman parte de la implementación de la evidencia científica derivada de investigación (29), y que son elementos importantes a la hora de ofrecer cuidado al paciente en la UCI (30).

\section{Conclusiones}

Describir la dimensión científico-clínica del cuidado de enfermería en la UCI de manera comparativa entre enfermeras de instituciones públicas y privadas permitió identificar elementos diferenciadores en la implementación de los procesos de atención de enfermería, administrativos e investigativos, derivados de la naturaleza propia de las instituciones, la heterogeneidad de las mismas y la conformación del equipo de enfermería participante que labora en la UCI, aspectos relacionados a su vez con su experiencia clínica y nivel de formación.

La evaluación de la dimensión científico-clínica mostró que algunas etapas del proceso de atención de enfermería se llevan a cabo de manera más rigurosa y completa por parte de las enfermeras de instituciones privadas, aunque las etapas del proceso administrativo e investigativo son implementadas de manera similar por las enfermeras del ámbito público y privado.

\section{Referencias}

1. Marshall JC, Bosco L, Adhikari NK, Connolly B, Diaz J V., Dorman T, et al. What is an intensive care unit? A report of the task force of the World Federation of Societies of Intensive and Critical Care Medicine. J Crit Care. 2017;37:270-6. http://dx.doi.org/10.1016/j.jcrc.2016.07.015

2. Parra M, Guaqueta S, Triana M. Perspectivas del cuidado de enfermería en UCI: una visión desde el modelo de cuidado HANC. Bogotá: Universidad Nacional de Colombia; 2013. 
3. Guaqueta S. Indicadores de la calidad del cuidado de enfermeria en las unidades de cuidado intensivo. Una mirada desde el modelo del cuidado de enfermeria en UCI: HANC. En: Avances en el cuidado de enfermería. Bogotá: Editorial Universidad Nacional; 2010. p. 299-315.

4. Castro M, Simian D. La enfermería y la investigación. Rev Médica Clínica Las Condes. 2018;29(3):301-10.

5. Hughes RG, editor. Patient safety and quality: an evidence-based handbook for nurses. Rockville, MD: Agency Healthc Res Qual US Dep Heal Hum Serv. 2008;(08):1-1403.

6. Rojas JG, Durango PP. Aplicación del proceso de atención de enfermería en Cuidados Intensivos. Invest. educ. enferm. 2010;28(3):323-35.

7. Soares MI, Terra F de S, Oliveira LS, Resck ZMR, Esteves AM da SD, Moura CDC. Nursing process and its application in an intensive care unit: integrative review. Rev Enferm UFPE line. 2013;7(5):4183-91.

8. Aires B, Moyano GB. Hacia un estado del arte en las perspectivas de desarrollo de competencias durante la formación de enfermería en cuidados intensivos. Rev Argentina Ter Intensiva. 2019;36(1):2-9.

9. Camelo $S$. Competencias profesionales de los enfermeros para trabajar en Unidades de Cuidados Intensivos: una revisión integradora. Rev Latino-Am Enferm. 2012;20(1):1-9.

10. Cortes J, Hernández M, Marchena T, Marqueti ML, Nava G. Estilos de liderazgo en jefes de servicio de enfermería. Rev Enfermería Neurológica. 2013;12(2):84-94.

11. Quintana-Zavala MO, Tinajero-González RM. Investigacion en enfermeria en unidad de cuidado intensivo adulto: una revision de literatura. Horiz Enferm. 2019;30(1):76-87.

12. Hanna ML, Villadiego MC. La administración de enfermería en el área clínica y sus implicaciones en el cuidado. Rev Colomb Enfermería. 2014;9(9):65-70.

13. Guaqueta-Parada S, Triana Restrepo M, Henao-Castaño AM. Validación del instrumento para medir la dimensión científico-clínica del cuidado de enfermería en UCI. Investigación en Enfermería Imagen y Desarrollo. [En prensa].

14. Lloret-Segura S, Ferreres-Traver A, Hernández-Baeza A, Tomás-Marco I. El análisis factorial exploratorio de los ítems: una guía práctica, revisada y actualizada. An Psicol. 2014;30(3):1151-69.

15. Henriques SE, Dos Santos Silva VL, Laus AM, Pedreschi Chaves LD. Perfil profissional de enfermeiros atuantes em unidades de terapia intensiva de um hospital de ensino Cienc y Enferm. 2013;(3):51-62.

16. Ministerio de Salud y Protección Social. Resolución Número 003100 de 2019 2019. p. 1-230. Disponible en: htt ps://www.minsalud.gov.co/Normatividad_Nuevo/Resoluci\%C3\%B3n\%20No.\%203100\%20de\%202019.pdf

17. Maurício LFS, Okuno MFP, Campanharo CRV, Lopes MCBT, Belasco AGS, Batista REA. Prática profissional do enfermeiro em unidades críticas: avaliação das characterísticas do ambiente de trabalho. Rev Lat Am Enfermagem. 2017;25. https://doi.org/10.1590/1518-8345.1424.2854

18. Reina N. El proceso de enfermeria: Instrumento para el cuidado. Umbral Científico. 2010;(17):18-23.

19. Rios C, Marcon del Sasso G. La tecnología: definiciones y reflexiones para la práctica en salud y enfermería. Texto Context - Enferm. 2008;17:1-2.

20. Murphy C, Merriman K, Zabka C, Penick M, Villamayor P. Patient-entered electronic healthcare records with electronic medical record integration: lessons learned from the field. CIN Computers, Informatics, Nursing. 2008; 26(5): 302.

21. Zamanzadeh V, Valizadeh L, Tabrizi FJ, Behshid M, Lotfi M. Challenges associated with the implementation of the nursing process: a systematic review. Iran J Nurs Midwifery Res. 2015;20(4):411-9.

22. Dal Sasso GTM, Barra DCC, Paese F, Almeida SRW de, Rios GC, Marinho MM, et al. Processo de enfermagem informatizado: metodologia para associação da avaliação clínica, diagnósticos, intervenções e resultados. Rev da Esc Enferm da USP. 2013;47(1):242-9.

23. Granero-Molina J, Fernández-Sola C, Peredo de Gonzales MH, Aguilera-Manrique G, Mollinedo-Mallea J, CastroSánchez AM. Proceso de enfermería: ¿qué significa para las enfermeras de Santa Cruz (Bolivia)? Rev da Esc Enferm da USP. 2012;46(4):973-9. 
24. Adamy EK, Zocche DA de A, Almeida M de A. Contribution of the nursing process for the construction of the identity of nursing professionals. Rev Gauch Enferm. 2020;41(spe):e20190143. https://doi.org/10.1590/1983 $-1447.2020 .20190143$

25. Dal Sasso GM, Barra DC. Cognitive workload of computerized nursing process in intensive care units. CIN Comput Informatics Nurs. 2015;33(8):339-45. https://doi.org/10.1097/CIN.0000000000000157

26. AL-Dossary RN, Kitsantas P, Maddox PJ. Residency programs and clinical leadership skills smong new saudi graduate nurses. J Prof Nurs. 2016;32(2):152-8. DOI: https://doi.org/10.1016/j.profnurs.2015.10.004

27. Carrillo A, Garcia L, Cardenas C, Diaz I, Yabrudy, N. La filosofía de Patricia Benner y la práctica clínica. Enfermería Glob. 2013;12(32):346-61.

28. Moreno-Casbas T, Fuentelsaz-Gallego C, González-María E, Gil de Miguel Á. Barreras para la utilización de la investigación. Estudio descriptivo en profesionales de enfermería de la práctica clínica y en investigadores activos. Enferm Clin. 2010;20(3):153-64. DOI: https://doi.org/10.1016/j.enfcli.2010.01.005

29. Ledesma ME, Rino MM. O Processo De Enfermagem Como Ações De Cuidado Rotineiro\#: construindo seu significado na perspectiva das enfermeiras assistencias. Perspective. Rev Lat Am Enfermagem [Internet]. 2009;17(3):328-334. https://doi.org/10.1590/S0104-11692009000300008

30. Llauradó-Serra M, Güell-Baró R, Castanera-Duro A, Sandalinas I, Argilaga E, Fortes-del Valle ML, et al. Barreras y motivaciones de los profesionales de enfermería para la utilización de la investigación en Unidades de Cuidados Intensivos y en el Servicio de Emergencias Médicas. Enferm Intensiva. 2016;27(4):146-54. DOI: https://doi. org/10.1016/j.enfi.2016.05.001

\section{Notas}

* Artículo original de investigación.

Licencia Creative Commons CC BY 4.0

Conflicto de intereses: Los autores declaran no tener ningún conflicto de intereses.

Financiación: Ninguna.

Cómo citar este artículo: Henao-Castaño A.M., Ávila M. L., Ramos Hernández S., Guaqueta-Parada S.R., Triana-Restrepo M.C. Evaluación de la dimensión científico-clínica del cuidado en el personal de enfermería. Investigación en Enfermería, Imagen y Desarrollo. 2021;23. https://doi.org/10.11144/Javeriana.ie23.edcc 